\title{
Estudo anatômico do ligamento patelofemoral lateral em joelho de cadáveres*
}

\author{
Anatomical study of the lateral patellofemoral \\ ligament in cadaver knees
}

Marcelo Schmidt Navarro ${ }^{1}$, Ricardo Dizioli Navarro², Jorge Akita Junior ${ }^{3}$, Moisés Cohen ${ }^{4}$

\section{RESUMO}

Objetivos: Descrever o ligamento patelofemoral lateral (LPFL) por meio de dissecação anatômica de joelhos de cadáveres frescos. Na literatura existe particular interesse pelo LPFL. Essa especial atenção é dada pela sua importância anatômica, biomecânica e pela necessidade da sua reconstrução nos casos de instabilidade medial da patela. Métodos: O LPFL foi estudado em 20 joelhos de 20 cadáveres frescos por meio de dissecações anatômicas. As dissecações foram padronizadas e os dados anotados enviados para análise estatística. $R \boldsymbol{e}$ sultados: O LPFL apresentou em média $16,05 \mathrm{~mm}$ de largura (desvio-padrão: 2,48), 42,10mm de comprimento (desvio-padrão: 8,84). Sua inserção femo-

* Trabalho realizado no Departamento de Ortopedia e Traumatologia da Escola Paulista de Medicina-Universidade Federal de São Paulo - UNIFESP - São Paulo (SP), Brasil.

1. Médico Ortopedista do Grupo de Traumatologia Esportiva da Disciplina de Ortopedia da Faculdade de Medicina do ABC FMABC - Santo André (SP), Brasil.

2. Livre-Docente, Professor Adjunto do Departamento de Ortopedia da UNIFESP - São Paulo (SP), Brasil.

3. Estagiário da Disciplina de Ortopedia da Faculdade de Medicina do ABC - FMABC - Santo André (SP), Brasil.

4. Livre-Docente, Professor Adjunto do Departamento de Ortopedia da UNIFESP - São Paulo (SP) - Brasil.

Endereço para correspondência: Marcelo Schmidt Navarro, Rua Dr. Henrique Calderazzo, 321, Centro de Estudos, 5o and., Bairro Paraíso - 09190-610 - Santo André (SP), Brasil. Tel.: 5511 6829-5000.

E-mail: msnavarro@uol.com.br

Recebido em 17/4/08. Aprovado para publicação em 24/6/08.

Copyright RBO2008 ral mostrou-se no epicôndilo femoral lateral e com expansão em forma de leque das suas fibras, predominantemente na região posterior e proximal ao epicôndilo lateral. Na patela a inserção encontrada foi na região súpero-lateral (na metade do terço superior no plano coronal), também com expansão das fibras, mas com menor intensidade. No plano sagital, o ligamento situava-se na metade posterior da borda da patela. $O$ LPFL foi isolado na segunda camada retinacular lateral, o retináculo transverso profundo. Conclusão: O LPFL situa-se na camada retinacular transversa profunda, com a presença de uma expansão das suas fibras em forma de leque, tanto na inserção femoral quanto na patelar. No epicôndilo femoral a inserção encontra-se mais posterior e proximalmente e na patela está na metade posterior da borda patelar (plano sagital) e no aspecto súpero-lateral.

Descritores - Ligamento patelar/anatomia \& histologia; Luxação patelar; Condromalácia da patela; Instabilidade articular; Dissecação

\section{ABSTRACT}

Objectives: To describe the lateral patellofemoral ligament (LPFL) after anatomical dissection of fresh cadaver knees. The literature shows a particular interest for the LPFL. Such special attention is due to its anatomical and biomechanical importance, and also due to the need to reconstruct the ligament in the cases of medial patellar instability. Methods: The LPFL was studied in 20 knees of 20 fresh cadavers by means of anatomical dissections. The dissections 
were standardized and the data were noted down and forwarded to statistical analysis. Results: The LPFL presented a mean $16.05 \mathrm{~mm}$ width (standard deviation of 2.48), $42.10 \mathrm{~mm}$ length (standard deviation of 8.84). The femoral insertion was found at the lateral femoral epicondyle and with a fan-like expansion of the fibers, predominantly in the posterior region proximal to the lateral epicondyle. In the patella, the insertion found was in the upperlateral region (in the middle of the upper third of the coronal plan), also with expanded fibers, but less intensely so. In the sagittal plan, the ligament was located in the posterior half of the border of the patella. The LPFL was isolated in the second lateral retinacular layer, the deep transverse retinaculum. Conclusion: The LPFL is located in the deep transverse retinacular layer with the presence of a fan-like expansion of its fibers both in the femoral and in the patellar insertions. The insertion is more posterior and proximal at the femoral epicondyle and in the posterior half of the patellar border (sagittal plan) and in the upper-lateral aspect.

Keywords - Patellar ligament/anatomy \& histology;
Patellar dislocation; Chondromalacia;
Patellae Joint instability; Dissection

\section{INTRODUÇÃO}

Os pacientes com afecções da articulação patelofemoral representam grande desafio aos ortopedistas, principalmente aos cirurgiões do joelho. Na literatura estão descritas mais do que 100 diferentes abordagens cirúrgicas para o tratamento da instabilidade patelar, que variam desde a mais simples, como a artroscopia, até as mais complexas, como as osteotomias femorais $^{(1-2)}$.

Procedimento bastante difundido e realizado muitas vezes indiscriminadamente, para o tratamento da instabilidade patelar, é a liberação retinacular lateral isolada, seja ela efetivada por via aberta ou artroscópica $^{(1)}$. A primeira descrição da liberação retinacular lateral na literatura foi reportada por Pollard e era combinada com a trocleoplastia femoral ${ }^{(3)}$. Todavia, o procedimento não foi mais descrito até ser novamente introduzido por Merchant et al $^{(4)}$ e popularizado artroscopicamente por McGinty et al ${ }^{(5)}$ e Metcalf ${ }^{(6)}$.

Hughston et $a^{(7)}$ foram os primeiros autores a apresentar a subluxação medial da patela como uma complicação da liberação retinacular lateral e descreveram a manobra de apreensão medial como parte da propedêutica dessa iatrogenia. Similarmente, diversos autores se preocuparam com a subluxação patelar medial. Podemos citar Nonweiler et al ${ }^{(8)}$, que também descreveram uma manobra, o teste de subluxação gravitacional. O paciente é colocado em decúbito lateral contralateral ao joelho afetado. O membro inferior afetado é passivamente abduzido com o quadríceps relaxado e o joelho estendido. Com isso, a gravidade desloca a patela medialmente e uma falha aparece ao longo da área onde foi realizada a liberação retinacular lateral. A patela pode ser passivamente colocada no sulco troclear pelo examinador através de uma pressão digital medial. Todavia, o paciente não consegue ativamente recolocar a patela no sulco pela contração voluntária do quadríceps, o que indica que o vetor de força lateral (músculo vasto lateral) foi comprometido no ato da liberação retinacular. Quando esse fato acontece, os autores consideram o teste de subluxação gravitacional positivo e significa que o músculo vasto lateral está destacado da patela e existe frouxidão do retináculo lateral. Propõem como tratamento para essa complicação a correção cirúrgica para restabelecer o ligamento patelofemoral lateral (LPFL) e o retináculo transverso profundo. No seguimento pós-operatório referiram que a sensação de instabilidade e o teste de subluxação gravitacional apresentaram-se negativos.

Adicionalmente, surgiram outros estudos que descreveram diversas técnicas cirúrgicas para a correção da subluxação medial. Como exemplo, encontramos o reparo ligamentar direto $^{(7-9)}$, a reconstrução do ligamento patelofemoral lateral ${ }^{(10-12)}$ ou a liberação retinacular medial artroscópica isolada ${ }^{(13)}$. Nessa última situação os autores referiram que, se houver falha na liberação medial, a reconstrução do ligamento patelofemoral lateral deve ser indicada.

O LPFL foi inicialmente citado na literatura nos estudos de Kaplan ${ }^{(14)}$. Ele desempenha papel estabilizador da patela e evita sua instabilidade medial ${ }^{(15)}$. Quan- 
do se apresenta muito tenso e encurtado, pode ocasionar dor anterior no joelho e instabilidade lateral da patela $^{(16)}$.

Segundo Dye et $a l^{(17)}$, com a melhora do conhecimento sobre a anatomia dos tecidos moles ao redor da patela, os cirurgiões ortopédicos devem estar aptos a prontamente identificar essas estruturas, melhor protegê-las e repará-las durante os procedimentos cirúrgicos. Portanto, com intuito de aprimorar nossos conhecimentos sobre a articulação patelofemoral (APF), fomos estimulados a desenvolver este trabalho. Através da dissecação de joelhos de cadáveres frescos, realizamos um estudo anatômico descritivo e comparativo sobre o LPFL. Adicionalmente, por meio da literatura, procuramos mostrar a importância do LPFL no estudo das patologias da APF.

\section{MÉTODOS}

No Serviço de Verificação de Óbitos da Capital (SVOC-FM-USP), realizamos a dissecação de 20 joelhos em 20 cadáveres. A pesquisa foi aprovada pelo Comitê de Ética em Pesquisa da UNIFESP (CEP 0093/ 07). Doze cadáveres eram do sexo masculino e oito do feminino. As peças eram preparadas a fresco e não formolizadas. Por meio da aplicação de um protocolo, identificamos as peças quanto ao sexo, idade, lado dissecado, data da dissecação, comprimento e largura do LPFL e descrição da inserção e origem do LPFL. Para medirmos esses valores, utilizamos um paquímetro milimetrado e alfinetes com a cabeça arredondada e colorida. Todas as medidas foram realizadas com o joelho em flexão de $30^{\circ}$. Se em algum momento fosse observada patologia ou cicatrizes no joelho, a peça era descartada. Portanto, os joelhos que apresentaram sinais de alguma patologia ou cicatrizes não foram utilizados. Quando ambos os joelhos apresentaram-se em boas condições de dissecação, a escolha pelo lado foi aleatória.

A média de idade dos cadáveres foi de 50,2 anos (variou de 20 a 88 anos). Dos 20 joelhos dissecados, 11 foram do lado direito e nove do esquerdo. Doze joelhos eram de cadáveres masculinos e oito femininos.

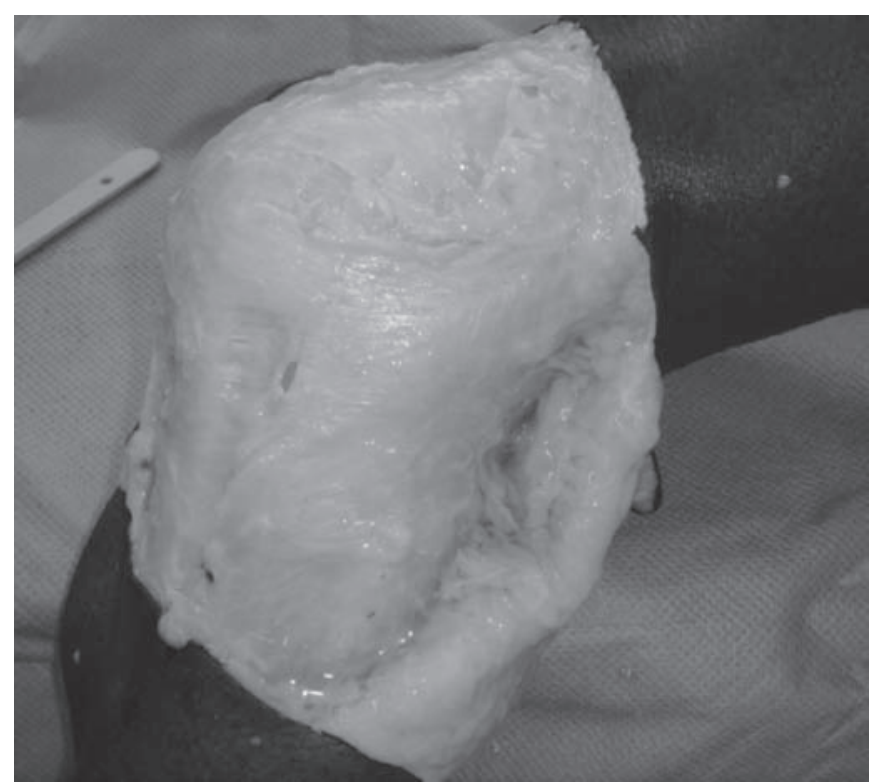

Figura 1 - Exposição da região ântero-lateral do joelho esquerdo após rebater-se a fáscia superficial

O cadáver foi posicionado em decúbito dorsal horizontal e um coxim foi colocado na região glútea ipsilateral para propiciar discreta lateralização do joelho. Dessa forma, a exposição das estruturas laterais durante o procedimento foi facilitada. Realizamos manobra de flexo-extensão repetidamente no joelho para removermos a rigidez cadavérica articular antes da dissecação.

Com um bisturi lâmina 22, iniciamos uma incisão ântero-lateral longitudinal no joelho na metade da distância entre o epicôndilo lateral e a borda lateral da patela, conforme descrito por Dye et al ${ }^{(17)}$. Após a incisão da pele e tecido celular subcutâneo, identificamos a fáscia superficial e a individualizamos do tecido celular subcutâneo. Dissecamos essa região superficial até expormos toda a face anterior da patela e o ligamento patelar. Com dissecação cuidadosa, rebatemos a fáscia superficial e identificamos o retináculo superficial oblíquo (figura 1). Esse retináculo foi minuciosamente removido e então pudemos identificar o retináculo transverso profundo (figura 2). Essas duas camadas da região lateral foram isoladas de acordo com os estudos de Fulkerson ${ }^{(18)}$.

Após a remoção da camada retinacular superficial, identificamos o LPFL. Apresentou-se como um espes- 


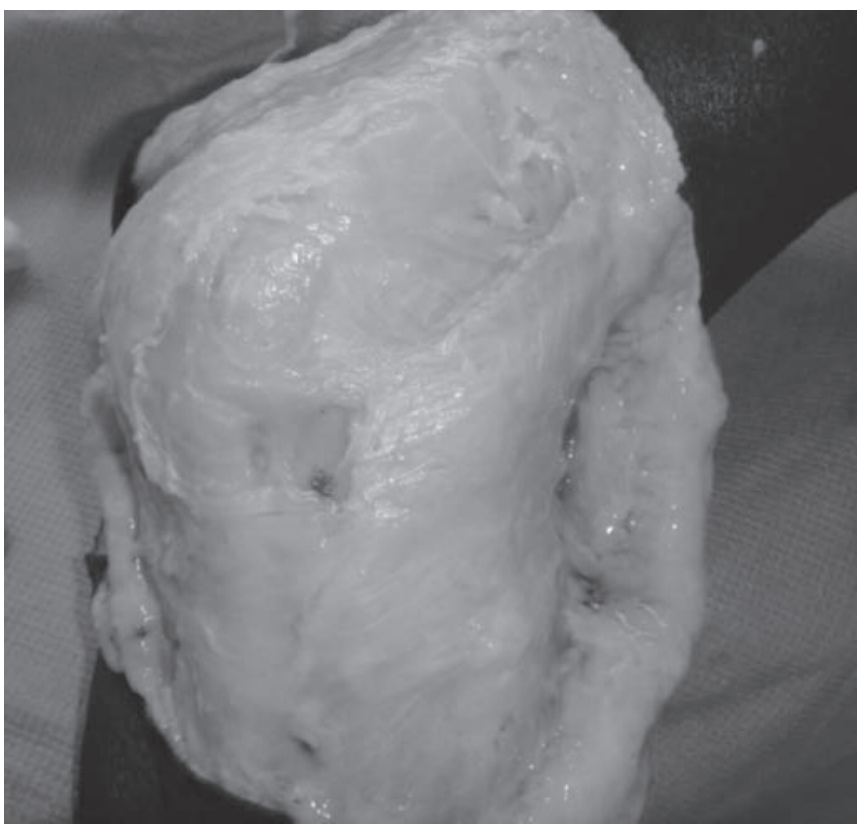

Figura 2 - Retináculo transverso profundo após a remoção da camada retinacular superficial

samento visível e palpável na camada do retináculo transverso profundo. Isolamos cuidadosamente o LPFL e o dissecamos até a região femoral e a patelar, anotando suas dimensões e descrevendo suas características. Para estudarmos sua inserção femoral, ressecamos cuidadosamente o trato iliotibial após sua desinserção no tubérculo de Gerdy (figura 3). Exatamente na junção entre o LPFL e o osso (fêmur ou patela), colocamos nossos alfinetes para medição do comprimento. A largura foi mensurada na metade da distância de seu comprimento.

Para a análise estatística utilizamos o programa SPSS (Statistical Package for Social Sciences) em sua versão 13.0 para a obtenção dos resultados.

\section{RESULTADOS}

Apresentamos os resultados obtidos nas nossas dissecações na tabela 1.

A largura média do LPFL foi de 16,05mm (de 13 a $20 \mathrm{~mm}$ ) com desvio-padrão de 2,48. O comprimento médio do LPFL apresentou 42,1 mm (de 31 a $53 \mathrm{~mm}$ ) com desvio-padrão de 8,84. Esses resultados estão expressos na tabela 2 .

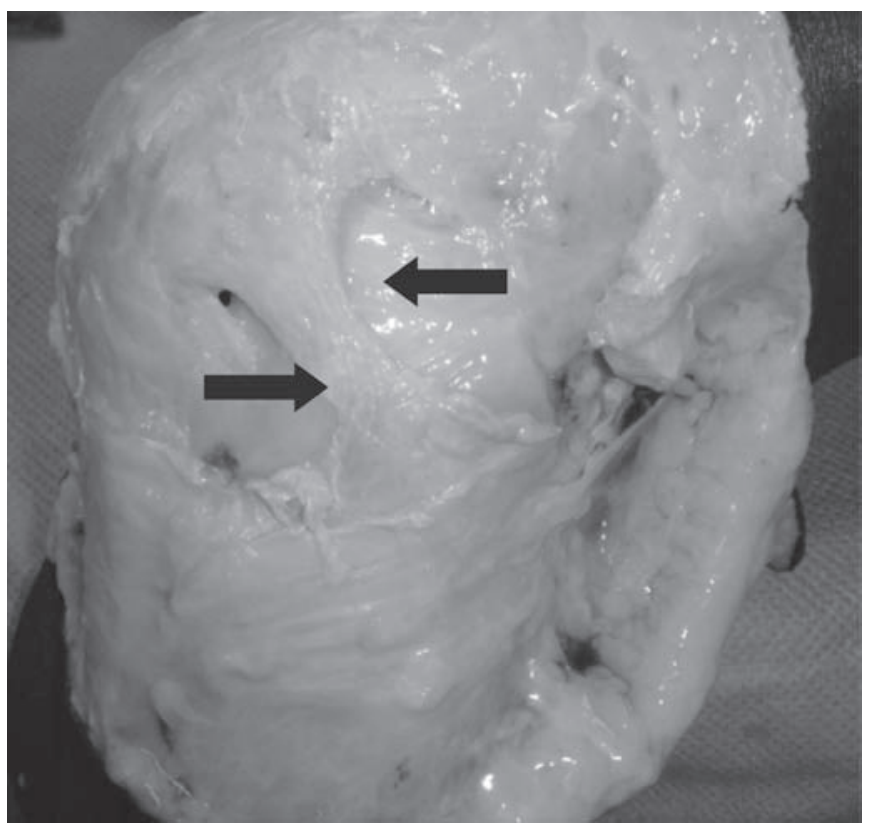

Figura 3 - LPFL em detalhe (setas) após a ressecção do trato iliotibial

TABELA 1

Resultados das dissecações do ligamento patelofemoral lateral no joelho de cadáveres frescos de acordo com número de ordem, sexo, idade (em anos), lado dissecado, LPFL_L (em mm) e LPFL_C (em mm)

\begin{tabular}{|c|c|c|c|c|c|}
\hline Número & Sexo & $\begin{array}{c}\text { Idade } \\
\text { (em anos) }\end{array}$ & Lado & $\begin{array}{c}\text { LPFL_L } \\
\text { (em mm) }\end{array}$ & $\begin{array}{l}\text { LPFL_C } \\
(\mathrm{em} \mathrm{mm})\end{array}$ \\
\hline 1 & $\mathrm{M}$ & 40 & $E$ & 13 & 31 \\
\hline 2 & $M$ & 20 & $\mathrm{D}$ & 18 & 38 \\
\hline 3 & $M$ & 60 & $\mathrm{D}$ & 14 & 32 \\
\hline 4 & $M$ & 50 & $\mathrm{D}$ & 20 & 58 \\
\hline 5 & $M$ & 25 & $E$ & 14 & 39 \\
\hline 6 & $M$ & 50 & $E$ & 16 & 49 \\
\hline 7 & $M$ & 42 & $E$ & 13 & 31 \\
\hline 8 & $M$ & 48 & $E$ & 19 & 48 \\
\hline 9 & $M$ & 60 & $\mathrm{D}$ & 14 & 45 \\
\hline 10 & $M$ & 43 & $D$ & 20 & 57 \\
\hline 11 & $M$ & 33 & $\mathrm{D}$ & 16 & 40 \\
\hline 12 & $M$ & 44 & $E$ & 14 & 54 \\
\hline 13 & $F$ & 61 & $\mathrm{D}$ & 13 & 31 \\
\hline 14 & $\mathrm{~F}$ & 88 & $\mathrm{D}$ & 14 & 34 \\
\hline 15 & $F$ & 71 & $E$ & 16 & 45 \\
\hline 16 & $\mathrm{~F}$ & 45 & $\mathrm{D}$ & 15 & 39 \\
\hline 17 & $\mathrm{~F}$ & 44 & $\mathrm{D}$ & 18 & 36 \\
\hline 18 & $\mathrm{~F}$ & 62 & $E$ & 18 & 37 \\
\hline 19 & $\mathrm{~F}$ & 57 & $E$ & 16 & 45 \\
\hline 20 & $\mathrm{~F}$ & 61 & $\mathrm{D}$ & 20 & 53 \\
\hline
\end{tabular}

M: masculino; F: feminino; D: direito; E: esquerdo; mm: milímetros; LPFL_L: largura do ligamento patelofemoral lateral em mm; LPFL_C: comprimento do ligamento patelofemoral lateral em mm.

Fonte: Serviço de Verificação de Óbitos da Capital - São Paulo 
TABELA 2

Resultado de todas as dissecações do LPFL considerando-se LPFL_L (em mm), LPFL_C (em mm), de acordo com as variáveis n, comprimento mínimo, comprimento máximo, comprimento médio e desvio-padrão

\begin{tabular}{lccccc}
\hline Variável & $\mathbf{n}$ & $\begin{array}{c}\text { Comprimento } \\
\text { Mínimo }\end{array}$ & $\begin{array}{c}\text { Comprimento } \\
\text { Máximo }\end{array}$ & $\begin{array}{c}\text { Comprimento } \\
\text { Médio }\end{array}$ & Desvio-padrão \\
\hline $\begin{array}{l}\text { LPFL_L } \\
\text { (em mm) }\end{array}$ & 20 & 13,00 & 20,00 & 16,05 & 2,48 \\
$\begin{array}{l}\text { LPFL_C } \\
\text { (em mm) }\end{array}$ & 20 & 31,00 & 58,00 & 42,10 & 8,84 \\
\hline
\end{tabular}

LPFL_L: largura do ligamento patelofemoral lateral em mm; LPFL_C: comprimento do ligamento patelofemoral lateral em $\mathrm{mm}$ $\mathrm{n}$ : quantidade de joelhos dissecada; mm: milímetros.

Fonte: Serviço de Verificação de Óbitos da Capital - São Paulo

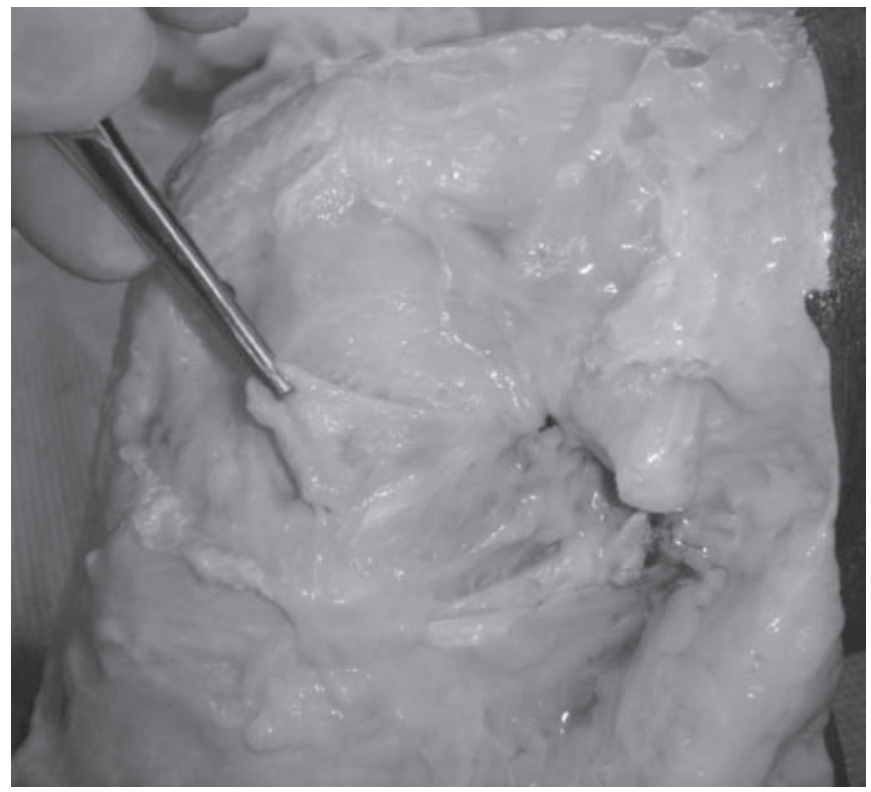

Figura 4 - Detalhe da inserção do LPFL no fêmur em forma triangular expansiva. Para fins didáticos, o LPFL foi seccionado.

O LPFL foi isolado na segunda camada retinacular lateral, o retináculo transverso profundo. Observamos LPFL como um espessamento proximal no retináculo transverso profundo e notamos que realiza a conexão entre o epicôndilo lateral e a região súpero-lateral da patela. Na inserção no epicôndilo lateral, o LPFL apresentou uma expansão das fibras em forma triangular, o que aumenta a sua área de ligação ao fêmur (figura 4). Apesar de sua inserção femoral estar localizada no epicôndilo lateral, a maioria de suas fibras está na região posterior e proximal ao epicôndilo.

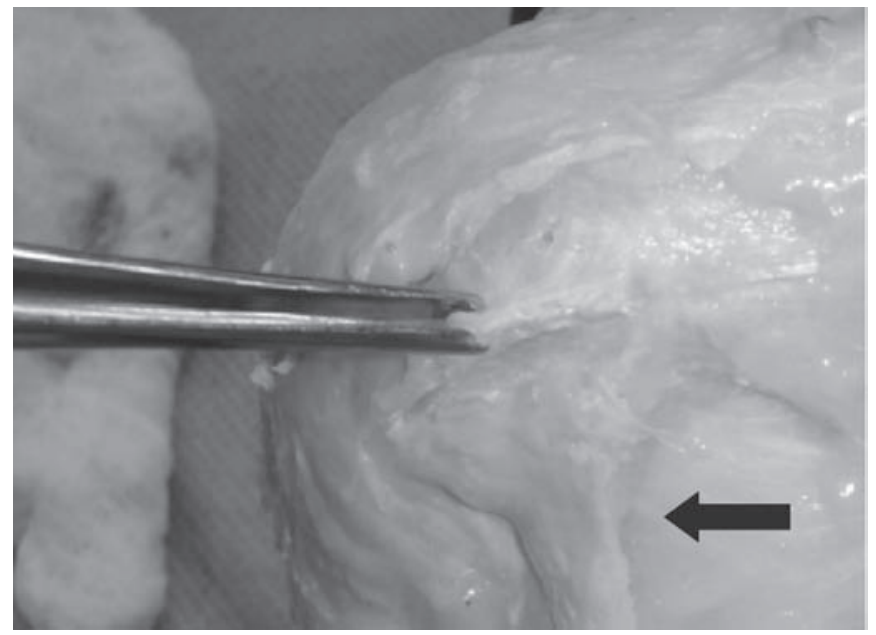

Figura 5 - Detalhe da inserção do LPFL na patela (seta)

Na patela, as fibras do LPFL inserem-se no aspecto súpero-lateral, especificamente na metade do terço superior no plano coronal. No plano sagital, a inserção ocorre na metade posterior da borda patelar. Também existe uma expansão em forma de leque das suas fibras similarmente à inserção femoral (figura 5).

\section{DISCUSSÃO}

Até os dias atuais, a dor e a instabilidade da articulação patelofemoral representam um grande desafio aos ortopedistas, principalmente aos cirurgiões de joe1 ho ${ }^{(8,19)}$. Diversos estudos investigaram a anatomia do joelho. A atual busca do conhecimento anatômico e biomecânico da articulação patelofemoral reflete o interesse dos investigadores em propiciar um tratamen- 
to eficiente para seus pacientes ${ }^{(17)}$. O estudo detalhado da anatomia do joelho é fundamental para o entendimento das patologias que o afetam ${ }^{(20-21)}$. Fulkerson ${ }^{(18)}$ observou que, para o tratamento dos atletas com dores na articulação patelofemoral, foi necessário um conhecimento meticuloso das estruturas retinaculares ao redor da patela.

Alguns autores incentivam os estudos sobre a anatomia do joelho, pois o aprendizado sobre a localização dos tecidos moles ao redor da patela capacita os cirurgiões a identificar as estruturas regionais para melhor protegê-las e repará-las durante os procedimentos cirúrgicos ${ }^{(17)}$. O conhecimento das camadas profundas do joelho pode aprimorar a abordagem e melhorar os resultados cirúrgicos dessa $\operatorname{articulação~}^{(22-23)}$. Tais observações nos estimularam a realizar um estudo anatômico no joelho humano que enfocou a articulação patelofemoral.

O retináculo lateral tenso e encurtado é citado em diversos estudos como uma das principais estruturas responsáveis pela instabilidade patelofemoral ${ }^{(24-26)}$. Julgamos que o LPFL também exerce papel fundamental na gênese das doenças patelofemorais, pois essa estrutura é considerada um componente do retináculo lateral e sua tensão exagerada pode ocasionar subluxação ou luxação lateral da patela ${ }^{(27)}$.

A partir de diversas constatações, observamos que a liberação retinacular lateral é muitas vezes indicada nos casos de tratamento cirúrgico para correção de algumas alterações patelofemorais, embora alguns autores acreditem que o procedimento não deve ser utilizado com freqüência ${ }^{(28)}$. Como complicação dessa liberação, podemos nos deparar com a subluxação patelofemoral medial, inclusive em sua forma oculta ${ }^{(29)}$. Essa entidade pode contribuir com os maus resultados obtidos após o procedimento de liberação retinacular lateral ${ }^{(30)}$. Dessa forma, a suspeita diagnóstica de subluxação medial iatrogênica da patela deve sempre estar presente no raciocínio do ortopedista, especialmente nos casos de resultados insatisfatórios. Para a correção da instabilidade medial é indicada a reconstrução ou reparação das estruturas retinaculares laterais (dentre elas, o LPFL) ou liberação retinacular medial ${ }^{(7-13,29)}$.
Em virtude da obrigação do conhecimento anatômico das estruturas retinaculares laterais do joelho na etiologia da instabilidade patelofemoral e da potencial necessidade de reconstrução do LPFL nos casos de instabilidade patelar medial, realizamos este estudo.

Não encontramos menção ao LPFL na Terminologia Anatômica $^{(31)}$. Seu primeiro registro na literatura foi feito por Kaplan ${ }^{(14)}$ como ligamento epicondilopatelar lateral. $\mathrm{O}$ autor não apresentou com detalhes as inserções femoral e patelar desse ligamento e nem o descreveu com minúcia. Fulkerson et al ${ }^{(32)}$ enfocaram maior atenção às estruturas laterais do joelho e descreveram o LPFL na mesma camada do retináculo transverso profundo. Nesse nível de dissecação, observaram que o LPFL estava inserido na região proximal do epicôndilo lateral femoral e que se conectava à região lateral da patela. Reider et $a^{(20)}$ nomearam o ligamento epicondilopatelar lateral descrito por Kaplan ${ }^{(14)}$ como ligamento patelofemoral lateral e afirmaram que é um espessamento palpável da cápsula articular e conecta a patela ao epicôndilo femoral. Teitge et $a^{(12)}$, no seu trabalho de reconstrução do LPFL, localizaram como ponto isométrico no fêmur o epicôndilo lateral e na patela o descreveram próximo à região anterior e na metade do terço superior da borda lateral. Não expuseram trabalhos anatômicos para a utilização desses pontos, mas empregam um isômetro para determinar os pontos isométricos. Feller et al ${ }^{(22)}$ e Andrikoula et $a l^{(33)}$ citaram o LPFL como componente do retináculo lateral transverso profundo, mas não o descrevem com maiores minúcias. Terry et al ${ }^{(23)}$ e $\mathrm{Amis}^{(34)}$ descreveram o LPFL como uma estrutura originada no aspecto profundo do trato iliotibial e que se insere na região lateral média da patela, mas não o expõem anatomicamente com mais detalhes. Vieira et al ${ }^{(21)}$ referiram que o LPFL é uma estrutura diferenciada e, quando seccionado, a patela excursiona medialmente. Segundo esses autores, tal fato indica que o LPFL atua na estabilidade patelar no plano sagital. Os autores também não especificaram com detalhes as inserções femoral e patelar do LPFL. Clarke et al ${ }^{(35)}$ descreveram três camadas na região lateral do joelho e consideraram o LPFL no mesmo nível do retináculo transverso profundo (segunda camada). 
Observamos nos trabalhos estudados que a localização anatômica do LPFL é descrita freqüentemente de forma controversa ou superficial e sua nomenclatura não está padronizada. Tal fato também nos motivou a realizar a investigação anatômica desse ligamento. Optamos pela terminologia ligamento patelofemoral lateral, utilizada pela primeira vez por Reider et $a l^{(20)}$.

De acordo com os diversos trabalhos anatômicos apresentados, o LPFL também pôde ser nitidamente isolado na camada retinacular lateral transversa profunda nas nossas dissecações. Acessamos essa camada após rebatermos o retináculo oblíquo superficial. Em todas as peças encontramos o LPFL. Situava-se profundamente ao trato iliotibial (TIT) e essas estruturas apresentaram-se intimamente relacionadas. Em três joelhos, todos do sexo masculino (casos 1, 7 e 9), encontramos uma intersecção muito intensa das fibras do LPFL e do TIT, o que exigiu grande cautela para sua separação. Observamos LPFL como um espessamento proximal no retináculo transverso profundo.

Concordamos com Fulkerson et al ${ }^{(32)}$ sobre a descrição da inserção do LPFL no fêmur (proximal ao epicôndilo lateral), mas observamos que ocorre uma expansão das fibras em forma de leque no local e existe também alguma inserção posterior ao epicôndilo. Teitge et $a l^{(12)}$, ao descreverem o ponto isométrico no fêmur do LPFL, durante sua reconstrução cirúrgica, o localizaram no epicôndilo femoral. Consideramos que, apesar de os autores descreverem tal localização, devemos ter ciência de que essa inserção não está apenas localizada em um ponto. Na literatura pesquisada só encontramos esse trabalho biomecânico que aborda a localização dos pontos isométricos na reconstrução do LPFL. Trata-se de uma nota técnica e os autores não expõem detalhadamente um estudo sobre o perfil e a evolução dos pacientes. Desse modo, acreditamos que estudos futuros nessa área serão necessários para maiores investigações.

Jeffreys ${ }^{(36)}$ observou uma banda anormal na borda súpero-lateral da patela a partir do trato iliotibial em pacientes portadores de luxação recidivante patelar. Vieira et $a l^{(21)}$ e Fulkerson et $a l^{(32)}$ acreditam que essa estrutura anormal possa ser o LPFL hipertrófico. Con- forme nossos achados anatômicos, essa possibilidade realmente pode ser considerada.

Outro fato importante que encontramos na literatura sobre a tensão retinacular lateral é que ela atua no desenvolvimento do joelho humano. Hinton et $a l^{(37)}$ sugeriram que as alterações anatômicas da instabilidade patelofemoral possam surgir em decorrência do aumento da tensão das partes moles ao redor da patela durante a evolução do indivíduo. Suspeitaram que a patela lateralizada durante o crescimento pode ser responsável pela morfologia de uma faceta patelar lateral mais larga. Adicionalmente, essas alterações anatômicas podem predispor à hipoplasia do côndilo femoral lateral, patela alta e sulco troclear raso. Essas anomalias podem gerar instabilidade na fase adulta. Acreditamos que tensão exagerada do LPFL durante o desenvolvimento do indivíduo pode ocasionar alterações na morfologia patelar na vida adulta e ter influência na gênese da instabilidade patelofemoral. Além disso, segundo Ficat et $a l^{(38)}$, a descompensação no equilíbrio entre o LPFL e o ligamento patelofemoral medial pode causar pressão lateral excessiva e originar condromalácia na faceta patelar lateral.

\section{CONCLUSÃO}

O ligamento patelofemoral lateral situa-se na camada retinacular transversa profunda, com a presença de uma expansão das suas fibras em forma de leque, tanto na inserção femoral quanto na patelar. No epicôndilo femoral a inserção encontra-se mais posteriormente e proximal e na patela está na metade posterior da borda patelar e no aspecto súpero-lateral. Apresentou em média 16,05mm de largura (desvio-padrão de 2,48) e $42,10 \mathrm{~mm}$ de comprimento (desvio-padrão de 8,84 ).

\section{AGRADECIMENTO}

Agradecemos ao colega Dr. Oswaldo Nascimento pelo auxílio e colaboração nas dissecações.

\section{REFERÊNCIAS}

1. Fithian DC, Paxton EW, Stone ML, Silva P, Davis DK, Elias DA, et al. Epidemiology and natural history of acute patellar dislocation. Am J Sports Med. 2004;32(5):1114-21.

2. Fulkerson JP. Biomechanics of the patellofemoral joint. In: Fulkerson JP. Disorders of the patellofemoral joint. 4th ed. Philadelphia: Lippincott Williams \& Wilkins; c2004. p. 24-42. 
3. Pollard B. Old dislocation of patella by intra-articular operation. Lancet. 1891;137:1203-4.

4. Merchant AC, Mercer RL. Lateral release of the patella. A preliminary report. Clin Orthop Relat Res. 1974;(103):40-5.

5. McGinty JB, McCarthy JC. Endoscopic lateral retinacular release: a preliminary report. Clin Orthop Relat Res. 1981; (158):120-5.

6. Metcalf RW. An arthroscopic method for lateral release of subluxating or dislocating patella. Clin Orthop Relat Res. 1982; (167):9-18.

7. Hughston JC, Deese M. Medial subluxation of the patella as a complication of lateral retinacular release. Am J Sports Med. 1988;16(4):383-8.

8. Nonweiler DE, DeLee JC. The diagnosis and treatment of medial subluxation of the patella after lateral retinacular release. Am J Sports Med. 1994;22(5):680-6.

9. Johnson DP, Wakeley C. Reconstruction of the lateral patellar retinaculum following lateral release: a case report. Knee Surg Sports Traumatol Arthrosc. 2002;10(6):361-3.

10. Abhaykumar S, Craig DM. Fascia lata sling reconstruction for recurrent medial dislocation of the patella. Knee. 1999;6(1): $55-7$.

11. Brinker MR, O'Connor DP, Flandry F, Hughston JC. Diagnosis and surgical correction of medial patellar subluxation. Oper Tech Sports Med. 2001;9(3):183-9.

12. Teitge RA, Torga Spak R. Lateral patellofemoral ligament reconstruction. Arthroscopy. 2004;20(9):998-1002.

13. Shannon BD, Keene JS. Results of arthroscopic medial retinacular release for treatment of medial subluxation of the patella. Am J Sports Med. 2007;35(7):1180-7.

14. Kaplan EB. Some aspects of functional anatomy of the human knee joint. Clin Orthop. 1962;23:18-29.

15. Andrish J. The biomechanics of patellofemoral stability. J Knee Surg. 2004;17(1):35-9.

16. Fulkerson JP, Arendt EA. Anterior knee pain in females. Clin Orthop Relat Res. 2000;(372):69-73.

17. Dye SF, Campagna-Pinto D, Dye CC, Shifflett S, Eiman T. Soft-tissue anatomy anterior to the human patella. J Bone Joint Surg Am. 2003;85(6):1012-7.

18. Fulkerson JP. Normal anatomy. In: Fulkerson JP. Disorders of the patellofemoral joint. 4th ed. Philadelphia: Lippincott Williams \& Wilkins; c2004. p. 1-23.

19. Fulkerson JP. Diagnosis and treatment of patients with patellofemoral pain. Am J Sports Med. 2002;30(3):447-56.

20. Reider B, Marshall JL, Koslin B, Ring B, Girgis FG. The anterior aspect of the knee joint. J Bone Joint Surg Am. 1981; 63(3):351-6.

21. Vieira EL, Vieira EA, da Silva RT, Berlfein PA, Abdalla RJ, Cohen M. An anatomic study of the iliotibial tract. Arthroscopy. 2007;23(3):269-74.

22. Feller JA, Amis AA, Andrish JT, Arendt EA, Erasmus PJ, Powers CM. Surgical biomechanics of the patellofemoral joint. Arthroscopy. 2007;23(5):542-53.
23. Terry GC, Hughston JC, Norwood LA. The anatomy of the iliopatellar band and iliotibial tract. Am J Sports Med. 1986; 14(1):39-45.

24. Larson RL, Cabaud HE, Slocum DB, James SL, Keenan T, Hutchinson T. The patellar compression syndrome: surgical treatment by lateral retinacular release. Clin Orthop Relat Res. 1978;(134):158-67.

25. Ishibashi Y, Okamura Y, Otsuka H, Tsuda E, Toh S. Lateral patellar retinaculum tension in patellar instability. Clin Orthop Relat Res. 2002;(397):362-9.

26. Aglietti P, Giron F, Cuomo P. Disorders of the patellofemoral joint. In: Insall JN, Scott WN. Surgery of the knee. 4th ed. New York: Churchill Livingstone; 2006. p. 807-936.

27. Luo ZP, Sakai N, Rand JA, An KN. Tensile stress of the lateral patellofemoral ligament during knee motion. Am J Knee Surg. 1997;10(3):139-44.

28. Christoforakis J, Bull AM, Strachan RK, Shymkiw R, Senavongse W, Amis AA. Effects of lateral retinacular release on the lateral stability of the patella. Knee Surg Sports Traumatol Arthrosc. 2006;14(3):273-7. Comment in: Knee Surg Sports Traumatol Arthrosc. 2007;15(6):825; author reply 826-7.

29. Fulkerson JP. Patellar tilt compression and the excessive lateral pressure syndrome. In: Fulkerson JP. Disorders of the patellofemoral joint. 4th ed. Philadelphia: Lippincott Williams \& Wilkins; c2004. p. 160-85.

30. Lattermann C, Toth J, Bach BR Jr. The role of lateral retinacular release in the treatment of patellar instability. Sports Med Arthrosc. 2007;15(2):57-60.

31. Sociedade Brasileira de Anatomia. Comissão de Terminologia Anatômica; Comissão Federativa da Terminologia Anatômica. Terminologia anatômica: terminologia anatômica internacional. São Paulo: Manole; 2001.

32. Fulkerson JP, Gossling HR. Anatomy of the knee joint lateral retinaculum. Clin Orthop Relat Res. 1980;(153):183-8.

33. Andrikoula S, Tokis A, Vasiliadis HS, Georgoulis A. The extensor mechanism of the knee joint: an anatomical study. Knee Surg Sports Traumatol Arthrosc. 2006;14(3):214-20.

34. Amis AA. Current concepts on anatomy and biomechanics of patellar stability. Sports Med Arthrosc. 2007;15(2):48-56.

35. Clarke HD, Scott WN, Insall JN, Pedersen HB, Math KR, Vigorita VJ, Cushner FD. Anatomy. In: Insall JN, Scott WN. Surgery of the knee. 4th ed. New York: Churchill Livingstone; 2006. p. 3-66.

36. Jeffreys TE. Recurrent dislocation of the patella due to abnormal attachment of the ilio-tibial tract. J Bone Joint Surg Br. 1963;45:740-3.

37. Hinton RY, Sharma KM. Patellar instability in childhood and adolescence. In: Insall JN, Scott WN. Surgery of the knee. 4th ed. New York: Churchill Livingstone; 2006. p. 1278-94.

38. Ficat RP, Philippe J, Hungerford DS. Chondromalacia patellae: a system of classification. Clin Orthop Relat Res. 1979;(144): 55-62. 